\section{Response to Peron et al.}

To the Editor: We thank Peron et al. ${ }^{1}$ for their interest and appreciation of our work ${ }^{2}$ and for their contribution to expanding the current limb copy-number-variation morbidity map. The authors describe a fetus with shortening and bowing of radius and ulna (mesomelic dysplasia). Using array comparative genomic hybridization the authors identified two de novo deletions flanking the $5^{\prime}$ and $3^{\prime}$ regions of the HOXD cluster (Supplementary Figure S1 online). The 5' centromeric $998-\mathrm{kb}$ deletion spans the region upstream of the $5^{\prime}$ HOXD genes including the genes ATF, ATP5G3, and KIAA1715 as well as the regulatory HOXD archipelago, a cluster of well-characterized limb enhancer elements (I-V, GCR, and Prox). ${ }^{3}$ The telomeric deletion is located downstream $3^{\prime}$ of the HOXD cluster (153 kb including MTX2). The authors conclude that the latter telomeric deletion is likely the cause of the phenotype via the loss of cis-regulatory elements controlling early $3^{\prime} H O X D$ genes in the proximal limb resulting in forelimb-specific mesomelic dysplasia. While the larger deletion removes all known 5' HOXD enhancers, we point out that the telomeric deletion does not include any of the previously characterized HOXD limb enhancers. Therefore, alternative explanations other than regulatory loss of function need to be considered.

Extensive mouse studies have revealed that during limb development, a subset of genes belonging to the HoxD cluster (Hoxd8-Hoxd13) are transcribed in two waves that determine the proximal-distal axis of the limb. The early wave is regulated by the forelimb enhancers CNS39 and CNS65 positioned in the telomeric topologically associating domain (TAD) activating Hoxd8-Hoxd11 transcription that patterns radius/tibia and ulna/fibula (Supplementary Figure S1). ${ }^{3}$ The succeeding wave is regulated by enhancers located in the centromeric TAD that activate Hoxd9-Hoxd13 expression to form the digits. ${ }^{4}$ Due to its strong chromatin interaction with the centromeric TAD, Hoxd13 is only very weakly contacted by the telomeric enhancers, leading to its absence of transcription in the developing radius/ulna region.

Several structural variants (SVs) at the locus in both mice and humans have been shown to disrupt this regulatory topography of the HOXD cluster causing limb malformation. The mouse mutant Ulnaless is caused by a 770-kb inversion of the HoxD cluster bringing Hoxd13 into the neighborhood of the telomeric enhancer elements and hijacking their regulatory potential. This results in the premature and ectopic onset of Hoxd13 transcription in proximal limb, where Hoxd13 is normally not expressed. This misexpression negatively interferes with the proliferation of mesenchymal cells in the zeugopod, which results in truncation of radius/tibia and ulna/fibula, with the forelimb being more severely affected. Tandem duplications of the HOXD cluster in humans cause Kantaputra mesomelic dysplasia, a severe mesomelic shortening of the upper limbs. These duplications place HOXD13 into the telomeric TAD $3^{\prime}$ regulatory landscape, thereby inducing misexpression of HOXD13 in the proximal limb, similar to the Ulnaless inversion.

Based on the specific upper-limb phenotype and the location of the deletions, we strongly suspect that the limb malformation reported by Peron et al. is not a result of a loss of function of anterior $3^{\prime}$ HOXD genes, because the $153-\mathrm{kb}$ deletion in the telomeric TAD does not include any known enhancer elements. Also, chromatin analyses revealed that the critical region for proximal limb enhancers in the telomeric TAD is not included into the reported deletion. ${ }^{3}$ Finally, the function of HOXD genes in developing limbs is largely redundant with that of HOXA genes and even a homozygous deletion of the entire murine HoxD cluster did not elicit any substantial mesomelic phenotype. Instead, we consider it more likely that the phenotype results from a gain of function of HOXD13 in proximal cells, as already reported in various cases in the mouse. Several scenarios are possible to account for this gain of function (Supplementary Figure S1).

First, the deletion of the centromeric regulatory landscape might allow ectopic interactions to be established between HOXD13 and the CNS39 and CNS65 enhancers located in the telomeric TAD. This particular mechanism of contact reallocation was previously observed in a mouse mutant where the centromeric regulatory landscape was displaced several $\mathrm{Mb}$ away from the $\operatorname{HoxD}$ cluster via a targeted inversion. Proximal limb bud cells showed ectopic expression of Hoxd13 and chromatin interactions between Hoxd13 and the telomeric landscape were increased significantly. These mice displayed a mild mesomelic dysplasia. ${ }^{3}$

Second, the presence of two deletions flanking both sides of the HOXD cluster might illustrate that the region between the two deletions containing the HOXD cluster itself is inverted in the affected individual, a condition that would remain unnoticed with the technology used by Peron et al. Such an inversion would place HOXD13 on the side of the telomeric regulatory elements resulting in proximal enhancer adoption and misexpression of HOXD13 with consequent development of severe mesomelic dysplasia. This situation would be similar to the Ulnaless allele in mouse and the Kantaputra duplication. This scenario is only possible if the deletions are located in cis on the same chromosome. Only quantitative reversetranscription polymerase chain reaction in combination with Sanger sequencing of the breakpoints will reveal the exact nature of this complex SV. Third, another possibility is that the centromeric deletion removes a boundary element at its centromeric end resulting in a fusion of the neighboring $\mathrm{TAD}$ 
with the $5^{\prime} H O X D$ genes leading to HOXD13 misexpression in the zeugopod.

In conclusion, SVs at the HOXD locus, including the case reported by Peron et al., demonstrate how complex rearrangements can reshuffle noncoding regulatory landscapes of developmental genes causing disease. A major shortcoming of our study and the report by Peron et al. is that array comparative genomic hybridization does not provide positional information nor does it detect inversions, smaller deletions, and duplications $(<20 \mathrm{~kb})$. However, as highlighted above, mapping of the exact breakpoints is essential to determine the true pathogenic potential of complex SVs. Whole-genome sequencing promises to be a more appropriate method to detect the full spectrum of SVs at single-nucleotide resolution, which will help to predict their effect on gene regulation. Moreover, in vivo mouse models using CRISPR-Cas9 system provide an excellent tool to understand the effect of chromosomal rearrangements in the genomic and chromatin regulatory context.

\section{SUPPLEMENTARY MATERIAL}

Supplementary material is linked to the online version of the paper at http://www.nature.com/gim

\section{DISCLOSURE}

The authors declare no conflict of interest.

\section{ACKNOWLEDGMENTS}

We thank Guillaume Andrey for helpful discussion. This work was supported by a grant from the Deutsche Forschungsgemeinschaft to M.S. (SP1532/2-1).

\section{Bjørt K. Kragesteen, $P h D^{1}$, Denis Duboule, $P h D^{2,3,4}$, Stefan Mundlos, $M D^{1}$ and Malte Spielmann, $M D^{5}$}

${ }^{1}$ Max Planck Institute for Molecular Genetics, Berlin, Germany; ${ }^{2}$ Department of Genetics and Evolution, University of Geneva, Geneva, Switzerland; ${ }^{3}$ School of Life Sciences, Federal Institute of Technology, Lausanne, Switzerland; ${ }^{4}$ Department of Genetics and Evolution, University of Geneva, Geneva, Switzerland; ${ }^{5}$ Department of Genome Sciences, University of Washington, Seattle, Washington, USA.

Correspondence: Malte Spielmann (spielman@uw.edu)

\section{REFERENCES}

1. Peron A, Boito S, Rizzuti T, et al. Prenatal upper-limb mesomelia and 2q31.1 microdeletions affecting the regulatory genome. Genet Med 2018;20:XXX-XXX.

2. Flöttmann R, Kragesteen BK, Geuer $S$, et al. Noncoding copy-number variations are associated with congenital limb malformation. Genet Med 2018:20:XXX-XXX.

3. Andrey G, Montavon T, Mascrez B, et al. A switch between topological domains underlies HoxD genes collinearity in mouse limbs. Science 2013;340:1234167.

4. Montavon T, Soshnikova N, Mascrez B, et al. A regulatory archipelago controls Hox genes transcription in digits. Cell 2011;147:1132-1145.

Advance online publication 1 March 2018. doi:10.1038/gim.2018.20 